\title{
气候变化和放牧活动对䊁隐子草种群的影响
}

\author{
汪诗平 王艳芬 陈佐忠 \\ (中国科学院植物研究所植被数量生态学重点实验室 北京 100093)
}

摘 要 䊁隐子草 (Cleistogenes squarrosa) 是一种多年生丛生小禾草, 属 $\mathrm{C}_{4}$ 植物, 在研究气候变化和放牧活动对草原 $\mathrm{C}_{4}$ 植物的影响方面是一种很好的指示植物。本文探讨了在气候变化及放牧干扰条件下䊁隐子草种群地上生物量、 种群特征和资源利用方式等方面的变化, 分析了 $\mathrm{C}_{4}$ 植物对气候变化的响应和在放牧干扰下的生态对策。结果表 明: 在气候温暖化的情况下 䊁隐子草种群生物量及其在群落中的比例都有所上升, 放牧大大加快了这一过程。通 过逐步回归分析得出糙隐子草种群最大地上生物量与生长季 (4 8 月) 总降雨量 $(r)$ 和平均温度 $(t)$ 的关系为 $: y=$ $-12.4511+0.0187 r+0.0601 t^{2}(p=0.003)$ 。总体上, 放牧明显降低了其种群高度和每丛生物量, 但显著提高了其 密度、盖度和地上生物量, 密度的变化受气候和放牧互作的影响。如果以植物光合所固定的 $100 \mathrm{~g}$ 的碳 (C) 所需要 的其它大量营养元素来衡量植物的养分利用效率, 则可以发现, 与其它主要植物如羊草 (Leymus chinensis) 相比 粘隐 子草对氮 $(\mathrm{N})$ 和硫 $(\mathrm{S})$ 的需求量最少 利用 $3.17 \mathrm{~g}$ 的 $\mathrm{N}$ 和 $0.31 \mathrm{~g}$ 的 $\mathrm{S}$ 就能生产 $100 \mathrm{~g}$ 的 C 而羊草则需要 $4.24 \mathrm{~g}$ 的 $\mathrm{N}$ 和 $0.41 \mathrm{~g}$ 的 $\mathrm{S}$ 才能生产 $100 \mathrm{~g}$ 的 $\mathrm{C} 。$ 说明在放牧使土壤比较贫痊的条件下䊁隐子草的养分利用效率较高, 这可能 是其在土壤比较贫痊的退化植物群落竞争中处于优势地位的又一重要原因。

关键词 气候变化 放牧 精隐子草 内蒙古典型草原

\section{EFFECT OF CLIMATE CHANGE AND GRAZING ON POPULATIONS OF CLEISTOGENES SQUARROSA IN INNER MONGOLIA STEPPE}

\author{
WANG Shi-Ping WANG Yan-Fen and CHEN Zuo-Zhong \\ ( Laboratory of Quantitative Vegetation Ecology , Institute of Botany, the Chinese Academy of Sciences , Beijing 100093 , China)
}

\begin{abstract}
Most rangelands in northern China are water-limited ecosystems. Even small changes in precipitation amount or season can affect the biological components that maintain nutrient, water and energy movement within and through these ecosystems. Inner Mongolia steppe is generally semi-arid with most of the precipitation coming during the summer from June to August. Land-management practices, such as livestock grazing, are affecting ecosystem structure and function and these activities may accelerate impacts of climate changes . Grazing-related changes to ecosystems become more pronounced as humans shift from nomadic or unrestricted practices of livestock management to practices that focus on human settlement. At the scale of a management unit (e.g. , watershed, allotment or paddock), precipitation amounts do not always correlate positively with vegetation productivity. Nutrient availability may strongly influence botanical composition and productivity. In general , spring and summer precipitation favors herbaceous vegetation that is more efficient in extracting water and will favor plants with $\mathrm{C}_{4}$ (warm-season) vs. $\mathrm{C}_{3}$ (cool-season) photosynthesis. The northern China steppes have few $\mathrm{C}_{4}$ plants. Little experimental work is being done in the field to examine the ecological impacts of climate change and grazing and their interactions on $\mathrm{C}_{4}$ plants in this region.

Cleistogenes squarrosa is a $\mathrm{C}_{4}$ plant. It is a short, perennial bunchgrass with drought tolerance, and tends to occupy medium degradation grassland in Inner Mongolia steppe. Therefore, it is a good indicator to study effect of climate change and grazing on $\mathrm{C}_{4}$ plants. In order to understand the responses of Cleistogenes squarrosa populations and ecological strategies to climate change and grazing, we monitored the data of climate (precipitation and temperature), $C$. squarrosa population and its community for 18 years under non-grazing conditions. The grazing (free grazing outside of fence) and non-grazing (fenced) experiment was conducted for 6 years. The nutrient efficiency ratios of the main herbages were evaluated in this study.

The experiment was conducted in the Leymus chinensis grassland of the Inner Mongolia Grassland Ecosystem Research Station located at $43^{\circ} 26^{\prime}-44^{\circ} 08^{\prime} \mathrm{N}, 116^{\circ} 04^{\prime}-117^{\circ} 05^{\prime} \mathrm{E}$ with elevation about $1000 \mathrm{~m}$ above sea level. The results showed that aboveground biomass and its proportion in the community increased with annual
\end{abstract}


mean temperature, and the regression equation between its aboveground biomass and mean temperature $(t)$ and total rainfall $(r)$ from April to August was : $y=-12.4511+0.0187 r+0.0601 t^{2}\left(\mathrm{R}^{2}=0.533, p=\right.$ $0.003)$. Generally, grazing significantly reduced plant height and aboveground biomass per bunchgrass , whereas its coverage, density and total aboveground biomass per square meter increased under grazing. Its nutrient efficiency ratios, such as $\mathrm{N}$ and $\mathrm{S}$, were higher compared with other dominant species, such as L. chinensis. $100 \mathrm{~g} \mathrm{C}$ produced required $3.17 \mathrm{~g} \mathrm{~N}$ and $0.31 \mathrm{~g} \mathrm{~S}$ for $C$. squarrosa, while $L$. chinensis population requires $4.24 \mathrm{~g} \mathrm{~N}$ and $0.41 \mathrm{~g} \mathrm{~S}$ under grazing. The high nutrient efficiency ratios maybe benefit $C$. squarrosa populations under competition with other species in the infertile soil.

Key words Climate change , Grazing, Cleistogenes squarrosa population , Inner Mongolia steppe

楉隐子草 (Cleistogenes squarrosa) 是一种多年生 丛生小禾草, 属 $\mathrm{C}_{4}$ 植物。由于草原上 $95 \%$ 以上的植 物均为 $\mathrm{C}_{3}$ 植物, $\mathrm{C}_{4}$ 植物种类很少(殷立娟等, 1997; 唐海萍, 1999)。唐海萍等 (2000) 认为, 在研究一个 功能群对环境变化的反应时, 可以用其中具有代表 性的一个或几个单独的物种反应来代表该功能群 (如 $\mathrm{C}_{3}$ 和 $\mathrm{C}_{4}$ 植物功能群)。而在中度放牧条件下或 退化草场经过一定的恢复阶段以后, 草场多演替为 以小禾草冷蒿 (Artemisia frigida) 为主的群落类型, 其 中 糙隐子草在群落中的重要性达 $20 \%$ 左右或更 大 , 其相对盖度达 30\% 40\% (王炜等, 1996 ;汪诗平 等,1998)。因而在研究气候变化和放牧活动对草原 $\mathrm{C}_{4}$ 植物的影响方面 楉隐子草种群的动态变化无疑 是一个很好的指示植物。

䊁隐子草草原群落广泛分布于欧亚草原区, 在 我国主要分布于内蒙古高原的典型草原地带, 多数 情况下为放牧演替类型。在内蒙古分布的总面积和 可利用面积达 867 万 $\mathrm{hm}^{2}$ 和 768 万 $\mathrm{hm}^{2}$, 分别占可利 用草地总面积的 $11 \%$ 和 $12 \%$; 同时，随着草原退化 的加剧, 其面积有不断扩大的趋势 (汪诗平等, 2001)。本文试图探讨 : 1) 气候变化对䊁隐子草种 群地上生物量的影响，2) 放牧对䊁隐子草种群地上 生物量和种群特征的影响;3) 䊁隐子草资源利用效 率。从而分析 $\mathrm{C}_{4}$ 植物对气候变化的响应和在放牧 干扰下的生态对策, 对于了解和认识在全球变化大 背景下放牧对羊草草原的影响提供科学的依据。

\section{1 材料和方法}

该试验在中国科学院内蒙古草原生态系统定位 研究站羊草样地进行, 有关试验区的基本生态环境 条件见陈佐忠等 (2000)。本试验所用的羊草围栏样 地是 1979 年建立的,此后每年进行群落动态的监 测。每年自 5 月初开始, 间隔 $15 \mathrm{~d}$ 齐地面则割 $1 \mathrm{~m}$ $\times 1 \mathrm{~m}$ 的样方, 每次重复 5 次, 直到 10 月初; 观测的 项目包括植株的高度、密度和盖度。然后在实验室
中分种烘干 $\left(65{ }^{\circ} \mathrm{C}\right)$ 称重。自 1991 年后 (1995 和 1996 年除外), 在围栏外 (自由放牧)对应的位置, 按 相同的方法取植物样品。本试验所用的气象资料为 $1981 \sim 1998$ 年的，气象资料为该站气象站所测。

1997 年 8 月，在羊草围栏样地外采集包括糙隐 子草在内的主要 8 种牧草, 用湿燃烧法、凯氏定氮法 和 $\mathrm{H}_{2} \mathrm{O}_{2}+\mathrm{HNO}_{3}(1: 4)$ 微波消化法分别测定植物的 C、 N、P 和 S 等元素含量, 其中, 用 ICP-AES 测定植物 全 $\mathrm{P}$ 和 $\mathrm{S}$ 的含量。

用统计软件的 ANOVA 和回归分析程序对数据 进行显著性检验和回归分析。

\section{2 结果与分析}

\section{1 气候变化}

表 1 和图 1 表明在近 20 年来,中国科学院内蒙 古草原生态系统定位研究站所在地区有变暖的趋 势。1981 1989 年年平均气温为 $0.0{ }^{\circ} \mathrm{C}$, 最冷月平 均气温为 $-22.3{ }^{\circ} \mathrm{C}$ 最热月平均气温为 $18.6{ }^{\circ} \mathrm{C}$, 极 端最低温度为 $-43.0{ }^{\circ} \mathrm{C}$ ( 1981 年), 年平均降水为 $334.6 \mathrm{~mm} ; 1990 \sim 1998$ 年年平均气温为 $1.0{ }^{\circ} \mathrm{C}$, 最冷 月平均气温为 $-21.13{ }^{\circ} \mathrm{C}$, 最热月平均气温为 19.0 ${ }^{\circ} \mathrm{C}$ 极端最低温度为 $-34.0{ }^{\circ} \mathrm{C}$ ( 1993 年), 年平均降 水为 $378.1 \mathrm{~mm}$ 。1990 1998 年与 $1981 \sim 1989$ 年相 比年平均气温显著升高了 $1.0{ }^{\circ} \mathrm{C}$, 其它指标虽然未 达到显著性水平, 但仍都有升高的趋势, 最冷月 (1 月)平均气温升高了 $1.2{ }^{\circ} \mathrm{C}$, 最热月 (7 月)平均气温 升高了 $0.4{ }^{\circ} \mathrm{C}$ 极端最低温度升高了 $9.0{ }^{\circ} \mathrm{C}$, 降水增 加了 $13 \%$ (表 1 , 图 2) ,年均气温每年极显著增加了 $0.12{ }^{\circ} \mathrm{C}$ (图 1)。主要体现在冬季增温上(表 1 ), 冬季 增温有可能使该地区春季的干旱进一步加剧。值得 一提的是, 经过单因子 $t$ 检验, 发现这 18 年间最冷 月平均气温、最热月平均气温、年平均气温和年降水 量均存在显著性 $(p<0.05)$ 或极显著性 $(p<0.01)$ 差 异, 说明年际间的波动很大(图 1 , 图 2)。另外, 还发 现年均气温与最冷月份 (1月) 的月均气温极显著相 关, 其回归方程为: $y=6.1765+0.2614 x(p=$ 
表 1 中国科学院内蒙古草原生态系统定位研究站 1981 1989 年与 1990 1998 年气温与降水的比较

Table 1 The comparison of temperature and precipitation between 1981-1989 and 1990-1998 at Inner Mongolia Grassland Ecosystem Research Station

\begin{tabular}{cccc}
\hline 年 Year & $1981 \sim 1989$ & $1990 \sim 1998$ & $p$ \\
\hline 年均气温 Annual mean temperature $\left({ }^{\circ} \mathrm{C}\right)$ & 0.0 & 1.0 & 0.022 \\
最冷月平均气温 Mean temperature in January $\left({ }^{\circ} \mathrm{C}\right)$ & -22.3 & -21.1 & 0.310 \\
最热月平均气温 Mean temperature in July $\left({ }^{\circ} \mathrm{C}\right)$ & 18.6 & 19.0 & 0.673 \\
极端最低温度 Lowest temperature $\left({ }^{\circ} \mathrm{C}\right)$ & $-43.0(1981)$ & $-34.0(1993)$ & $/$ \\
年均降水 Annual mean precipitation $(\mathrm{mm})$ & 334.6 & 378.1 & 0.202 \\
\hline
\end{tabular}

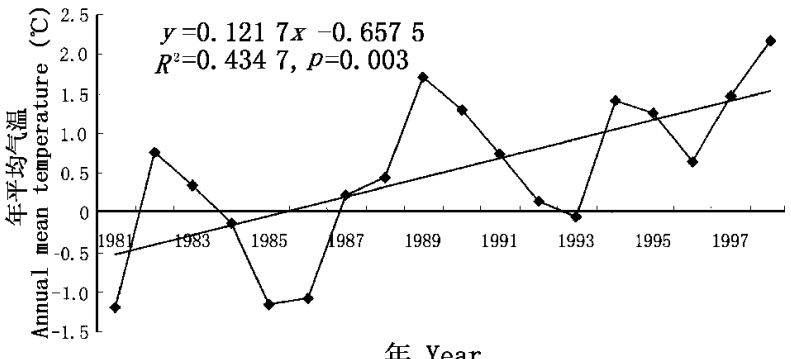

年 Year

图 $11981 \sim 1998$ 年年均气温的变化 (图中直线为趋势线)

Fig. 1 The dynamics of mean temperature from 1982 to 1998 (The straight line is trend line)

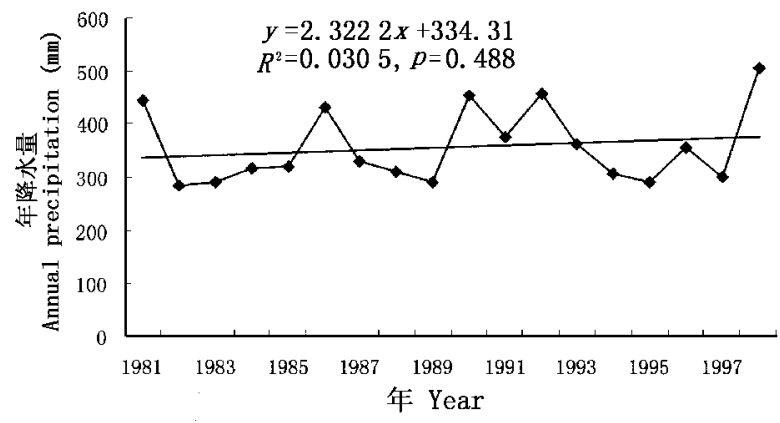

图 $21981 \sim 1998$ 年年降水量动态 (图中直线为趋势线)

Fig. 2 The dynamics of annual precipitation from 1981 to 1998 (The straight line is trend line)

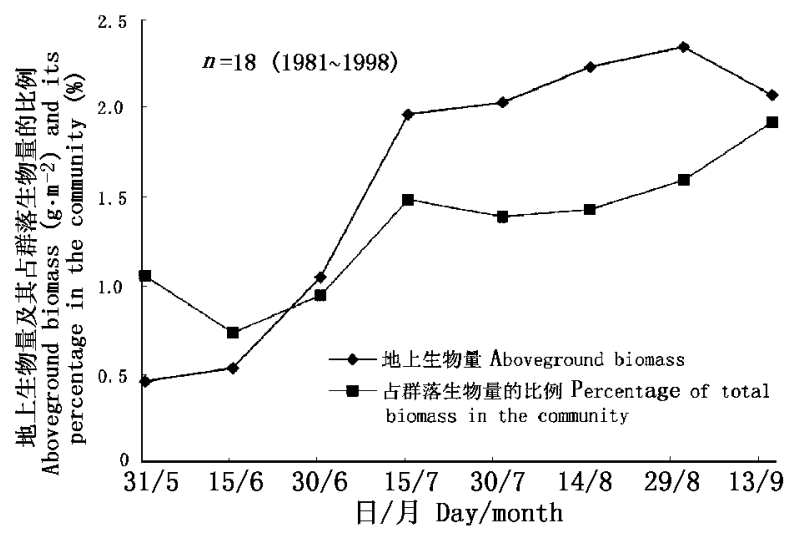

图 3 䊁隐子草种群地上生物量及其在群落中 比例的季节变化 (1981 1998)

Fig. 3 The dynamics of aboveground biomass and its percentage in the community of Cleistogenes squarrosa population with growing season from 1981 to 1998

$0.005)$, 这里,$y$ 为年均气温 $\left({ }^{\circ} \mathrm{C}\right), x$ 为 1 月份气温 $\left({ }^{\circ} \mathrm{C}\right)$ 。
2.2 糙隐子草种群地上生物量季节和年度变化

18 年的平均结果表明 (图 3) ,䊁隐子草种群地 上生物量在 7 月 15 日以前快速增长, 在 8 月中下旬 达到最大值 其在群落中的比例也有类似的趋势, 但 在 7 月中旬到 8 月下旬，其相对比例保持相对稳定， 而 9 月份以后其比例有所升高, 说明其凋落枯萎过 程可能比其它植物慢, 因为在该地区 9 月初已开始 下霜 植物基本停止生长。糙隐子草种群地上生物 量随生长天数的回归方程为: $y=0.5077+0.0512 x$ $-0.0002 x^{2}(p=0.001)$,由此可见, 当其生长 $123 \mathrm{~d}$ (即 8 月中下旬) 时达到地上最大生物量。

通过 18 年粮隐子草种群最大地上生物量的观 测 发现基本上可以以 1989 年为界, 1989 年以前, 该 种群最大地上生物量有下降的趋势 (图 4) , 其原因 可能是 1979 年围栏以前，该地区为自由放牧地，虽 然草地没有出现明显的退化，但放牧仍抑制了高大 禾草如羊草和大针茅 (Stipa grandis) 等的生长(李永 宏，1988; $\mathrm{Li}, 1989)$ 然而，当围栏牧压消除以后，这些 高大禾草迅速恢复, 在光、水和其它资源的竞争中占 有优势, 使得粮隐子草种群的绝对生物量和相对比 例都有所下降。而 1989 年以后, 其最大地上生物量 及其比例则有上升的趋势, 可能主要与气温升高和 降水增加等有关。一般地, $\mathrm{C}_{4}$ 植物较 $\mathrm{C}_{3}$ 植物更适 应高温环境 (方精云等, 2000)。糙隐子草种群最大 地上生物量与年均气温高度正相关 $\left(R=0.543^{* *}, p\right.$ $<0.01$ ), 其回归方程为: $y=3.1919-0.7675 x-$ $0.4055 x^{2}+0.9190 x^{3}(p=0.009)$; 与 $4 \sim 8$ 月份的总 降雨量也高度正相关 $\left(R=0.505^{* *}, p<0.01\right)$, 其回 归方程为: $y=16.8610-0.1043 x+0.0002 x^{2}(p=$ $0.006)$, 但与年降水量相关不显著 $(p>0.05)$ 。通过 逐步回归分析得出糙隐子草种群最大地上生物量与 生长季 (4 8 月)平均温度和总降雨量的关系为: $y$ $=-12.4511+0.0187 r^{* *}+0.0601 t^{2 * *}\left(R^{2}=\right.$ $0.533, p=0.003)$, 这里, $y$ 为鈢隐子草种群最大地 上生物量 , $r$ 为 $4 \sim 8$ 月份总降雨量 $(\mathrm{mm}), t$ 为 $4 \sim 8$ 月份平均气温。 


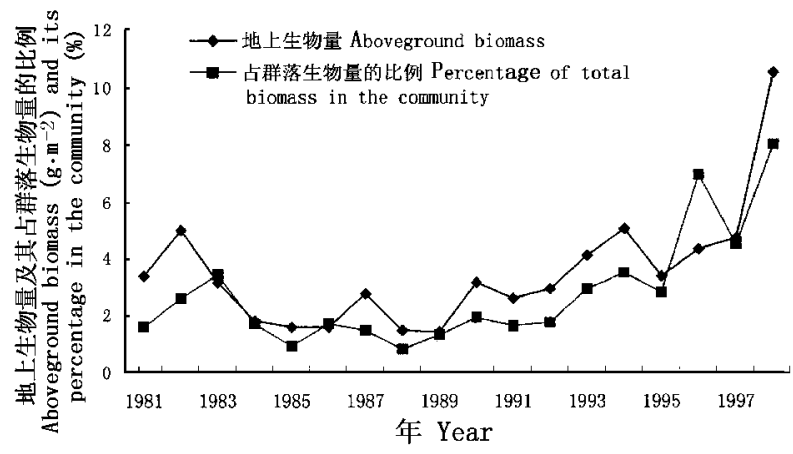

图 4 糙隐子草种群地上最大生物量及其在群落 中的比例的年际变化

Fig.4 The dynamics of aboveground maximum biomass and its percentage in the community of Cleistogenes squarrosa population from 1981 to 1998

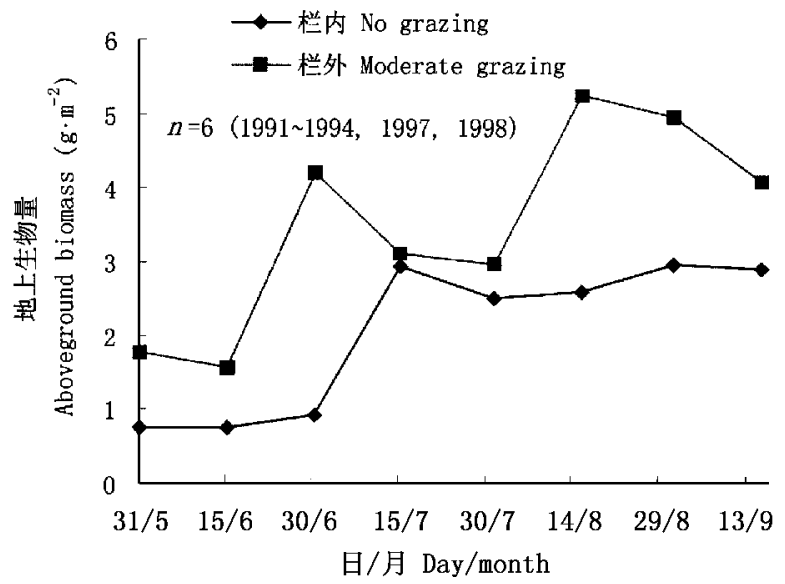

图 5 放牧对粘隐子草种群地上生物量 (均值) 的影响 (1991 1994,1997, 1998)

Fig.5 Effect of grazing on aboveground biomass of Cleistogenes squarrosa population (Mean of 1991 to 1994,1997 and 1998)

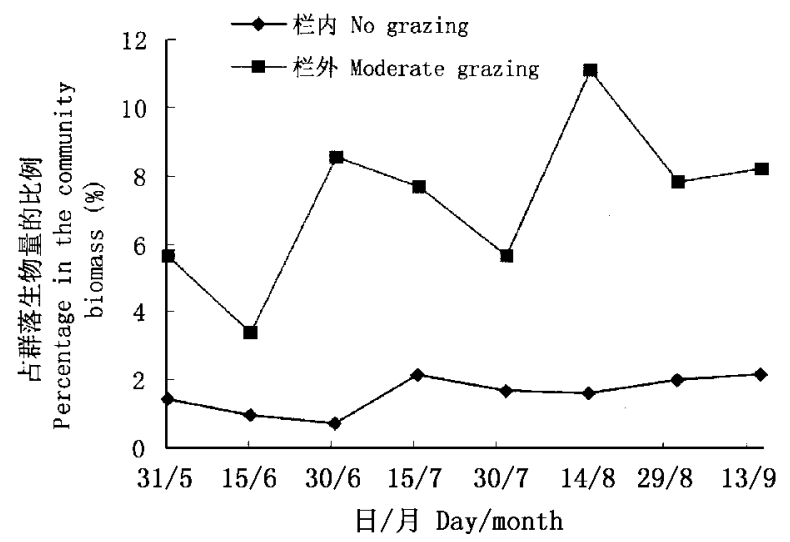

图 6 放牧对䊁隐子草种群地上生物量占群 落总生物量比例的影响 (6 年的均值)

Fig.6 Effect of grazing on percentage of Cleistogenes squarrosa population in the community (Mean of 1991 to 1994,1997 and 1998)

\section{3 放牧对糙隐子草种群的影响}

通过 6 年(1991 1994, 1997 和 1998 年)的观测
表明，由于放牧家畜自由采食活动抑制了羊草、大针 茅等高大植物层片的生长, 从而为低矮的植物腾出 了更多的空间资源，有利于粗隐子草等小禾草的生 长发育。与围栏内相比, 整个生长季节围栏外粗隐 子草种群地上现存量明显较高 $(p<0.05)$ (图 5), 虽然 7 月份差异不大，但栏外未计算家畜所采食的 量 根据以前的研究发现, 放牧绵羊喜食粘隐子草 (汪诗平，2000，2001），因此，如果考虑放牧家畜的采 食量，则差异更大。另外，在 7 月份水热同期, 适度 放牧可能促进了䊁隐子草种群的补偿性生长 (汪诗 平等, 2001)。围栏内, 整个季节糙隐子草种群地上 生物量占群落总量的比例不超过 $2 \%$, 然而在栏外， 其比例增加了 3～5 倍, 甚至达到 11\%（图 6) ;如果 考虑放牧家畜的选择性采食，其比例可能更高。

从图 7 可以看出，在 1997 和 1998 年，栏外糙隐 子草种群地上现存量占整个群落的比例高达 $25 \%$ 左右，显著高于栏内 $(p<0.01)$, 而 $1991 \sim 1994$ 年， 差异不显著的原因仍然可能是未考虑放牧家畜的采 食量所致。

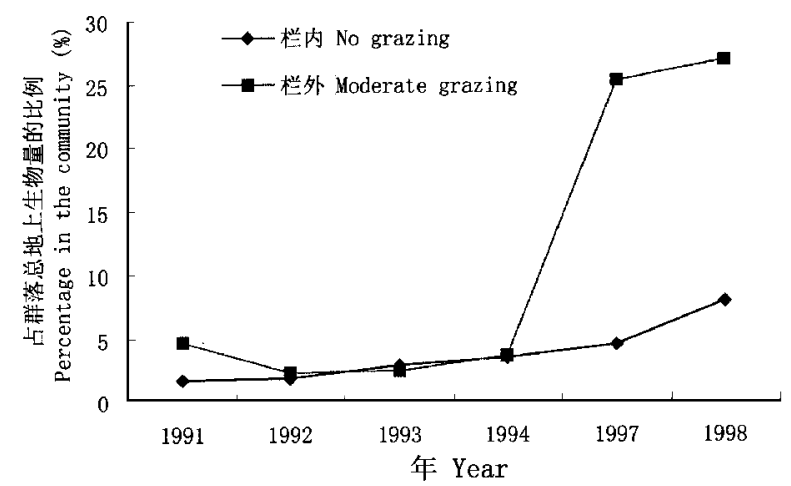

图 7 不同年份放牧对䊁隐子草种群占群落 总地上生物量比例的影响

Fig.7 Effect of grazing on the percentage of aboveground biomass of Cleistogenes squarrosa population in the community under different years

\section{4 放牧对䊁隐子草种群特征的影响}

总体上,放牧显著降低了植物的高度和每丛生 物量, 而提高了种群的盖度、密度和单位面积内的地 上生物量 (表 2)。但不同年份放牧的影响仍有差 异, 对放牧较敏感的种群特征为植株高度、密度和每 丛生物量, 在大多数年份都对放牧产生明显的反应。 通过统计检验, 除密度指标以外均不存在年度与放 牧处理间的互作作用 $(p>0.05)$, 说明密度不仅受放 牧的影响, 而且年度间差异较大, 即气候变化的影响 也很显著。

\section{5 不同植物养分利用策略}


从表 3 可以看出,除菊叶委陵菜 (Potentilla tanacetifolia) 外,其它植物 C 含量差异不大,均在 $45 \%$ 左右 ;泠蒿和小叶锦鸡儿( Caragana microphylla) 全 $\mathrm{N}$ 含量较高, 但以糙隐子草的含量最低;除冷蒿 外，主要植物全 $\mathrm{P}$ 的含量差异不大，而全 $\mathrm{S}$ 的含量仍 以冷蓄最高, 羊草的次之, 大针茅的最低 (表 3)。如 果以植物光合所固定的 $100 \mathrm{~g}$ 的 C 所需要的其它大 量营养元素来衡量植物的资源利用效率, 则可以发 现, 在栗钙土羊草草原上, 冷蒿对资源的利用效率最
低，如生产 $100 \mathrm{~g}$ 的 $\mathrm{C}$, 则需要 $5.54 \mathrm{~g}$ 的 $\mathrm{N} 、 0.48 \mathrm{~g}$ 的 $\mathrm{P}$ 和 $0.58 \mathrm{~g}$ 的 $\mathrm{S}$;小叶锦鸡儿次之;而糙隐子草对 $\mathrm{N}$ 的需求量最少，利用 $3.17 \mathrm{~g}$ 的 $\mathrm{N}$ 就能生产 $100 \mathrm{~g}$ 的 $\mathrm{C}$ 对 $\mathrm{P}$ 的需求量相差不大 (冷莒除外) , 对 $\mathrm{S}$ 的需求 量也较低 (大针茅和菊叶委陵菜除外)。说明在放牧 使土壤比较贫痊的条件下, 糙隐子草可以利用较少 的养分资源量进行同样的光合产物 (碳) 的生产, 即 其较高的养分利用效率可能是其在土壤比较贫痊的 群落竞争中处于优势地位的又一重要原因。

表 2 放牧对䊁隐子草种群特征的影响 (1997 年 8 月中旬)

Table 2 The influence of grazing on characteristics of Cleistogenes squarrosa population in the middle of August , 1997

\begin{tabular}{|c|c|c|c|c|c|c|}
\hline $\begin{array}{l}\text { 年 } \\
\text { Year }\end{array}$ & $\begin{array}{c}\text { 处理 } \\
\text { Treatment }\end{array}$ & $\begin{array}{l}\text { 高度 } \\
\text { Height } \\
(\mathrm{cm})\end{array}$ & $\begin{array}{l}\text { 盖度 } \\
\text { Coverage } \\
(\%)\end{array}$ & $\begin{array}{c}\text { 密度 } \\
\text { Density } \\
\left(\text { cluster } \mathrm{m}^{-2} \text { ) }\right.\end{array}$ & $\begin{array}{c}\text { 地上生物量 } \\
\text { Aboveground } \\
\text { biomass }\left(\mathrm{g} \mathrm{m}^{-2}\right)\end{array}$ & $\begin{array}{c}\text { 每丛地上生物量 } \\
\text { Aboveground biomass } \\
\text { per cluster ( } \mathrm{g} \mathrm{cluster}^{-1} \text { ) }\end{array}$ \\
\hline \multirow[t]{2}{*}{1991} & G & $6.7 \pm 0.6^{\mathrm{a}}$ & $3.0 \pm 1.6^{\mathrm{a}}$ & $12.3 \pm 6.9^{\mathrm{b}}$ & $3.8 \pm 3.2^{c}$ & $0.26 \pm 0.11^{\mathrm{a}}$ \\
\hline & NG & $11.0 \pm 2.6^{\mathrm{a}}$ & $1.0 \pm 0.7^{\mathrm{a}}$ & $3.3 \pm 2.5^{\mathrm{a}}$ & $1.0 \pm 1.6^{\mathrm{a}}$ & $0.33 \pm 0.23^{\mathrm{a}}$ \\
\hline \multirow[t]{2}{*}{1992} & G & $7.3 \pm 1.5^{\mathrm{a}}$ & $2.1 \pm 1.2^{\mathrm{a}}$ & $5.3 \pm 3.4^{\mathrm{a}}$ & $2.0 \pm 1.3^{\mathrm{a}}$ & $0.38 \pm 0.07^{\mathrm{a}}$ \\
\hline & NG & $11.7 \pm 5.0^{\mathrm{a}}$ & $1.2 \pm 0.8^{\mathrm{a}}$ & $2.0 \pm 0.8^{\mathrm{a}}$ & $1.2 \pm 0.9^{\mathrm{a}}$ & $0.58 \pm 0.20^{\mathrm{a}}$ \\
\hline \multirow[t]{2}{*}{1993} & G & $8.3 \pm 0.6^{\mathrm{a}}$ & $2.8 \pm 2.3^{\mathrm{a}}$ & $12.3 \pm 6.1^{\mathrm{b}}$ & $2.1 \pm 2.1^{\mathrm{a}}$ & $0.17 \pm 0.12^{\mathrm{a}}$ \\
\hline & NG & $14.7 \pm 1.2^{\mathrm{b}}$ & $1.7 \pm 0.6^{\mathrm{a}}$ & $3.3 \pm 2.3^{\mathrm{a}}$ & $2.1 \pm 2.5^{\mathrm{a}}$ & $0.64 \pm 0.15^{\mathrm{c}}$ \\
\hline \multirow[t]{2}{*}{1994} & G & $4.7 \pm 0.6^{\mathrm{a}}$ & $2.8 \pm 2.4^{\mathrm{a}}$ & $16.3 \pm 6.4^{\mathrm{b}}$ & $1.7 \pm 2.8^{\mathrm{a}}$ & $0.07 \pm 0.02^{\mathrm{a}}$ \\
\hline & NG & $14.3 \pm 2.9^{\mathrm{b}}$ & $2.9 \pm 2.0^{\mathrm{a}}$ & $4.0 \pm 2.4^{\mathrm{a}}$ & $2.3 \pm 5.3^{\mathrm{a}}$ & $0.58 \pm 0.50^{c}$ \\
\hline \multirow[t]{2}{*}{1997} & G & $5.0 \pm 1.7^{\mathrm{a}}$ & $13.0 \pm 2.7^{\mathrm{c}}$ & $31.2 \pm 4.3^{\mathrm{c}}$ & $8.2 \pm 5.2^{b}$ & $0.26 \pm 0.15^{\mathrm{a}}$ \\
\hline & NG & $9.3 \pm 3.1^{\mathrm{a}}$ & $1.3 \pm 0.7^{\mathrm{a}}$ & $2.8 \pm 0.4^{\mathrm{a}}$ & $2.1 \pm 1.2^{\mathrm{a}}$ & $0.87 \pm 0.26^{\mathrm{b}}$ \\
\hline \multirow[t]{2}{*}{1998} & G & $6.0 \pm 1.0^{\mathrm{a}}$ & $6.0 \pm 3.8^{\mathrm{a}}$ & $28.0 \pm 7.8^{\mathrm{c}}$ & $14.0 \pm 7.1^{\mathrm{b}}$ & $0.50 \pm 0.09^{\mathrm{a}}$ \\
\hline & NG & $11.0 \pm 5.6^{\mathrm{b}}$ & $3.0 \pm 1.2^{\mathrm{a}}$ & $4.0 \pm 2.0^{\mathrm{a}}$ & $4.4 \pm 2.2^{\mathrm{a}}$ & $1.10 \pm 0.25^{\mathrm{b}}$ \\
\hline 总平均 & G & $6.4 \pm 2.0^{\mathrm{a}}$ & $5.2 \pm 4.8^{c}$ & $16.4 \pm 10.4^{c}$ & $5.6 \pm 2.6^{b}$ & $0.34 \pm 0.11^{\mathrm{a}}$ \\
\hline Mean & NG & $12.1 \pm 3.7^{\mathrm{c}}$ & $1.7 \pm 1.3^{\mathrm{a}}$ & $3.1 \pm 2.2^{\mathrm{a}}$ & $2.0 \pm 1.2^{\mathrm{a}}$ & $0.65 \pm 0.37^{\mathrm{b}}$ \\
\hline
\end{tabular}

只进行同一年放牧 $(\mathrm{G})$ 与不放牧 (NG)间相同项目的比较，不同字母表示差异显著或极显著 Significant tests were only carried out between grazing $(\mathrm{G})$ and no grazing $(\mathrm{NG})$ treatments in the same year, a and $\mathrm{b}(p<0.05)$ and a and $\mathrm{c}(p<0.01)$

表 3 不同植物 C、N、P 和 $S$ 元素含量及其比例

Table 3 The concentration of $\mathrm{C}, \mathrm{N}, \mathrm{P}$ and $\mathrm{S}$ and their ratio

\begin{tabular}{lccccc}
\hline \multicolumn{1}{c}{ 植物名 Species } & $\mathrm{C}(\%)^{*}$ & $\mathrm{~N}(\%)^{*}$ & $\mathrm{P}(\%)^{*}$ & $\mathrm{~S}(\%)^{*}$ & $\mathrm{C}: \mathrm{N}: \mathrm{P}: \mathrm{S}$ \\
\hline 䊁隐子草 Cleistogenes squarrosa & 46.30 & 1.47 & 0.15 & 0.14 & $100: 3.17: 0.32: 0.31$ \\
羊草 Leymus chinensis & 45.53 & 1.93 & 0.12 & 0.19 & $100: 4.24: 0.27: 0.41$ \\
大针茅 Stipa grandis & 46.64 & 1.82 & 0.12 & 0.10 & $100: 3.89: 0.27: 0.21$ \\
冰草 Agropyron cristatum & 43.77 & 1.64 & 0.14 & 0.16 & $100: 3.74: 0.32: 0.39$ \\
草地早熟禾 Poa pratensis & 44.65 & 1.74 & 0.13 & 0.17 & $100: 3.89: 0.30: 0.39$ \\
冷藁 Artemisia frigida & 45.86 & 2.54 & 0.22 & 0.27 & $100: 5.54: 0.48: 0.58$ \\
菊叶委陵菜 Potentilla tanacetifolia & 35.17 & 1.24 & 0.10 & 0.11 & $100: 3.54: 0.29: 0.30$ \\
小叶锦鸡儿 Caragana microphylla & 45.31 & 2.37 & 0.13 & 0.18 & $100: 5.23: 0.29: 0.39$ \\
\hline
\end{tabular}

* 干物质百分比 Percentage of dry matter

\section{3 讨 论}

尽管对于当代气候变化有不同的认识,也存在 截然相反的现象,但全球性气候变暖的事实在最近 10 多年中得到了广泛的认同。据全球地表气温的 最新分析表明(方精云等,2000)，在过去 100 多年 中, 全球地表气温平均上升了 $0.6{ }^{\circ} \mathrm{C}$ 。一般而言 北
半球高纬度地区比低纬度地区增温快;而另一方面， 冬季温暖化现象比夏季明显。根据我们自己近 20 年的气象监测也表明有类似的结果，如内蒙古典型 草原地区, 夏季温度变化不明显, 而冬季增温较大, 如 20 世纪 90 年代较 80 年代平均增加 $1.0{ }^{\circ} \mathrm{C}$ (表 1) (陈佐忠等,2000)。另一方面, 该地区气候变化的另 一特点是年均降水量有增加的趋势, 如 20 世纪 90 
年代较 80 年代年平均增加 $43.5 \mathrm{~mm}$ 左右(表 1),20 世纪 80 年代的 8 年中只有一年的降水量超过平均 值 $(350 \mathrm{~mm}$ ) ,而在 20 世纪 90 年代, 9 年中有 5 年超 过了均值。这种气候变化必将对该地区的植被产生 广泛而深远的影响。

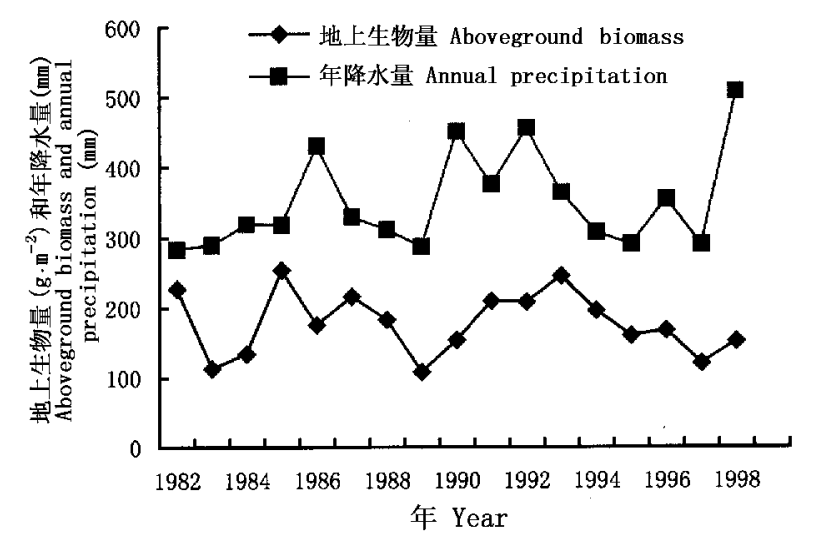

图 8 不同年份羊草草原地上生物量动态变化

Fig. 8 The dynamics of aboveground biomass of Leymus chinensis community under no grazing from 1982 to 1998

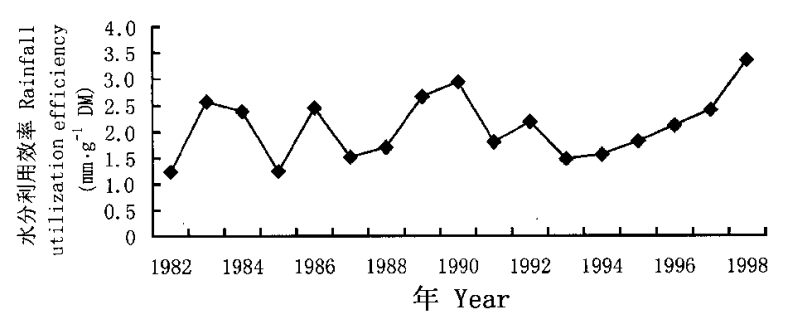

图 9 羊草草原不同年份水分利用效率的变化

Fig.9 The rainfall utilization efficiency of Leymus chinensis community from 1982 to 1998

一般认为, 在半干旱地区, 增温和降水量的增加 将有利于该地区的植物生长, 但由于增温主要发生 在冷季, 因而可能加剧了春旱的发生; 同时, 草原地 区绝大多数植物为 $\mathrm{C}_{3}$ 植物, 温度的升高对其的生长 将产生不利的影响(方精云等,2000)。另外, 草原植 物是与当地气候长期协同进化的产物, 由于不同植 物或植物功能群对气候变化的响应不同，因而也导 致群落总生物量的较大变异。图 8 表明, 在自然状 态下, 羊草草原地上生物量的年度动态变化基本上 与年降水量多少的变化相一致。17 年的平均地上 生物量为 $(178.1 \pm 45.2) \mathrm{g} \mathrm{m}^{-2}$, 其变异系数为 25 . $4 \%$, 而 17 年平均降水量为 $(351.2 \pm 69.6) \mathrm{mm}$, 其变 异系数为 $19.8 \%$ 。值得一提的是 地上生物量的变 化并非与降水量的变化完全一致,如 1986、1990、 1992 和 1998 年，其降水量分别达 430.0、450.3、455. 0 和 $507.0 \mathrm{~mm}$, 但相应的地上生物量只有 175.5 、
153.9、208.5 和 $152.0 \mathrm{~g} \mathrm{~m}^{-2}$, 甚至低于平均水平。 这主要与草原植物生长的生态适宜度有关 (李镇清 等, 1997) 即当植物生长季降水量超过草原植物生 长需要时, 水分不再是第一限制性因子, 而光照和积 温则成为限制植物生长的第一因子，降水量或云量 的增加将导致光照的不足，从而降低了水分的利用 效率, 17 年羊草草原平均水分利用效率为 1.97 $\mathrm{mm} \mathrm{g} \mathrm{g}^{-1} \mathrm{DM}$, 而上述 4 个年份均大于此值, 尤其以 1998 年水分利用效率最低，每生产 $1 \mathrm{~g} \mathrm{DM}$ 消耗 3.34 $\mathrm{mm}$ 的水(图 9)。因此年降水量特别是生长季内的 降水量及水热的相对平衡可能是影响草原植物生长 的最关键因子。

研究结果表明(汪诗平等，2001），在适当放牧条 件下 䊁隐子草种群明显的存在 超补偿性生长”。 就个体而言, 适牧可以刺激䊁隐子草个体地上净光 合效率; 但在高强度牧食下 楉隐子草个体则通过降 低地下生产力的生产来达到地上较低的补偿性生 产。就种群而言 䊁隐子草的 超补偿性生长” 主要 是形成小株丛和高密度的种群结构以适应较贫痊的 生境条件(表 2)。因为, 在自然条件下, 高大禾草的 遮荫不仅降低了䊁隐子草的光能可利用性, 而且还 改变了到达冠层以下的光线中红光/远红光 $(R / F R)$ 的比例 (Casal et al. , 1987)。当光线通过植物冠层 时 红光比远红光减弱得更快, 即到达冠层以下的光 线中 $\mathrm{R} / \mathrm{FR}$ 的比例比自然光线中的低。许多可控实 验证明, R/FR 比例的降低可减少植株的分薛 (Deregibus et al ., 1985 ; Casal et al ，1987)。因此, 在自然群落中 楉隐子草高度较低, 主要是对光资源 的竞争。而在放牧条件下, 放牧抑制了高大禾草, 一 方面可能是改善了到达楉隐子草冠层的光线质量 ; 另一方面, 可能也与由于草群高度和盖度的降低, 导 致地表温度较高有关。

养分利用效率”已被广泛地用于评价植物对养 分需要和利用能力上 (Gourley et al . , 1994)。尽管 有关 养分利用效率” 的概念很多, 但一些人将它表 述为 每单位养分吸收量所生产的总植物生物量” (Baligar et al ., 1990 ; Godwin \& Blair , 1991)。本研 究以植物光合每生产 $100 \mathrm{~g}$ 碳所需的资源量作为衡 量养分利用效率的指标, 认为在有限或痊薄的土壤 养分资源条件下，对养分能更有效利用或具有更大 利用能力的物种可能在竞争中处于优势。由于放牧 往往导致土壤养分的贫痊化（关世英等，1997），这可 能也是䊁隐子草对放牧适应的另一种生态策略 (表 3)。 


\section{参 考 文 献}

Baligar, V. C., R. R. Duncan \& N. K. Fageria. 1990. Soilplant interactions on nutrient use efficiency in plants. In: Baliger, V. C. \& R. R. Duncan eds. Crops as enhancers of nutrient use. San Diego: Academic Press. $351 \sim 373$.

Casal, J. J., R. A. Sanchez \& V. A. Deregibus. 1987. Tillering responses of Lolium multiflorum plants to changes of $\mathrm{R} / \mathrm{FR}$ ratio typical of sparse canopies. Journal of Experimental Botany, 38: $1432 \sim 1439$.

Chen, Z. Z. (陈佐忠) \& S. P. Wang (汪诗平). 2000. Chinese steppe ecosystem. Beijing: Science Press. 412. (in Chinese)

Deregibus, V. A., R. A. Sanchez, J. J. Casal \& M. J. Trlica. 1985. Tillering responses to environment of red light beneath the canopy in a humid natural grassland. Journal of Applied Ecology, 22: $199 \sim 206$.

Fang, J. Y. (方精云), Y. H. Tang (唐艳鸿), J. D. Lin (林 俊达) \& G. M. Jiang (蒋高明). 2000. Global ecology-climate change and ecological responses. Beijing: China Higher Education Press. 319. (in Chinese with English abstract)

Godwin, D. C. \& G. J. Blair. 1991. Phosphorus efficiency in pasture species. V. A comparison of white clover accessions. Australian Journal of Agriculture Research, 42: $531 \sim 540$.

Gourley, C. J. P., D. L. Allan \& M. P. Russelle. 1994. Plant nutrient efficiency: a comparison of definitions and suggested improvement. Plant and Soil, 158: 29 37 .

Guan, S. Y. (关世英), P. Q. Qi (齐沛钦), S. A. Kang (康 师安) \& J. B. Chang (常进宝). 1997. Effects on the steppe soil nutrient contents under different grazing intensities. Research on Grassland Ecosystem (草原生态系统研究), 5: 16 22 . (in Chinese)

Li, Y. H. 1989. Impact of grazing on Leymus chinensis steppe and Stipa grandis steppe. Acta Oecologica/Oecologica Application, 10: $31 \sim 46$.

Li, Y. H. (李永宏). 1988. The divergence and convergence of Aneurolepidium chinensis steppe and Stipa grandis steppe under the grazing influence in Xilin river valley, Inner Mongolia. Acta Phytoecologica et Geobotanica Sinica (植物生态学与地植物学 学报), 12: 189 196. (in Chinese with English abstract)

Li, Z. Q. (李镇清) \& J. Z. Ren (任继周). 1997. The fitness model and its application of rangeland plant. Chinese Journal of
Ecology (生态学杂志), 16(3): 71 75. (in Chinese with English abstract)

Tang, H. P. (唐海萍) . 1999. Distribution of $\mathrm{C}_{4}$ plants along the Northeast China Transect (NECT), and its correlation to the environmental factors. Chinese Science Bulletin (科学通报), 44 : $416 \sim 420$. (in Chinese)

Tang, H. P. (唐海萍) \& G. M. Jiang (蒋高明). 2000. Plant functional type and its significance in ecological research. Chinese Journal of Applied Ecology (应用生态学报), 11：461 464. (in Chinese with English abstract)

Wang, S. P. (汪诗平) \& Y. F. Wang (王艳芬). 2001. The study on over-compensation growth of Cleistogenes squarrosa population in Inner Mongolia steppe. Acta Botanica Sinica (植物学 报), 43: $413 \sim 418$. (in Chinese with English abstract)

Wang, S. P. (汪诗平), Y. H. Li (李永宏), Y. F. Wang (王 艳芬) \& Y. H. Han (韩苑鸿). 1998. The succession of Artemisia frigida rangeland and multivariation analysis under different stocking rates in Inner Mongolia. Acta Agrestia Sinica (草 地学报), 6: 299 305. (in Chinese with English abstract)

Wang, S. P. (汪诗平). 2000. The dietary composition of fine wool sheep and plant diversity in Inner Mongolia steppe. Acta Ecologica Sinica (生态学报), 20:951 957. (in Chinese with English abstract)

Wang, S. P. (汪诗平). 2001. The relationship between dietary diversity and range plant diversity of Artemisia frigida community under different stocking rates. Acta Ecologica Sinica (生态学 报), 21:237 243. (in Chinese with English abstract)

Wang, S. P. (汪诗平), C. G. Wan (万长贵) \& R. E. Sosebee. 2001. The influence of different defoliational intensities and light qualities (ratio of $\mathrm{R} / \mathrm{FR}$ ) on vegetative reproduction of Lovegrass. Chinese Journal of Applied Ecology (应用生态学 报), 12: $245 \sim 248$. (in Chinese with English abstract)

Wang, W. (王炜), Z. L. Liu (刘忠龄), D. Y. Hao (郝敦 元) \& C. Z. Liang (梁存柱) . 1996. Research on the restoring succession of the degenerated grassland in Inner Mongolia. II. Analysis of the restoring processes. Acta Phytoecologica Sinica (植物生态学报)，20: 460 471. (in Chinese with English abstract)

Yin, L. J. (殷立娟) \& P. Wang (王萍). 1997. Distribution of $\mathrm{C}_{3}$ and $\mathrm{C}_{4}$ photosynthetic pathways of plants on the steppe of Northeastern China. Acta Ecologica Sinica (生态学报), 17: $112 \sim 123$. (in Chinese with English abstract) 